\title{
Foliation of the Schwarzschild black hole surrounded by quintessence
}

Siddiqui A.A., Zafar S.

Department of Mathematics, School of Natural Sciences, National University of Sciences and Technology, Islamabad, Pakistan;

E-mail: Siddiqui<azad@sns.nust.edu.pk>;

In this paper we have first derived the compactified Kruskal-Szekeres like coordinates for the metric describing the Schwarzschild spacetime surrounded by quintessence and have provided maximally extended Carter-Penrose diagram for the geometry. We have then obtained a foliation of the Carter-Penrose diagram of this spacetime by the hypersurfaces having constant mean extrinsic curvature.

Keywords: foliation, quintessence, mean extrinsic curvature.

DOI: $10.18698 / 2309-7604-2015-1-473-482$

\section{Introduction}

In order to model behavior of the universe as a whole, we apply general relativity. To do this, we usually make some far-reaching assumptions. For example, the universe is isotropic or homogeneous etc. Evidently, matter distribution is highly irregular (non homogeneous) on small scales but on large scale it looks more and more uniform (homogeneous). So we have good physical background to study simple cosmological models with the assumption of homogeneity and isotropy of the universe. This led to the cosmological principle, which states that, the universe looks the same at all times to all observers [1]. The concept of a moment of time is ambiguous in general relativity and is replaced by a time slice or three dimensional spacelike hypersurface. So in order to define a globally accepted time parameter, we slice up (foliate) the spacetime by a sequence of disjoint spacelike hypersurfaces. Hence a particular time means a particular spacelike hypersurface. The hypersurfaces, $t=$ constant, may be constructed in a number of ways and there is no preferred way of slicing and consequently no preferred time. Maximal slicing (foliation by hypersurfaces of zero mean extrinsic curvature) of the Schwarzschild spacetime was tried by A. Lichnerowicz [2] but was unsuccessful. York defined a time parameter which is proportional to the mean extrinsic curvature $(K)$, called the York time [3]. So one may expect foliation of a spacetime by hypersurfaces having constant mean extrinsic curvature. Brill et. al. [4] have provided a comprehensive discussion of foliation of static, spherically symmetric spacetimes by hypersurfaces of constant mean extrinsic curvature (named as $K$-surfaces), but were unsuccessful to completely foliate the Schwarzschild spacetime. They used only the non negative values of $K$ and proposed that a use of full range of values might provide with a complete foliation. A complete 
foliation of the Schwarzschild geometry is provided in [5] and foliation of the Ressiner Nordstrom spacetime in [6]. In the following subsections, we first discuss the black hole surrounded by the quintessence (SHQ), construct the Kruskal-Szekeres like coordinates for this geometry, and then present the foliation of SHQ by hypersurfaces of constant mean extrinsic curvature.

\section{Schwarzschild Black Hole Surrounded by Quintessence}

In this section we briefly introduce the Schwarzschild black hole surrounded by quintessence as derived by V. V. Kiselev [7]. This derivation assumes the gravitational field to be spherically symmetric and static; and the energy momentum tensor of the form

$$
\begin{gathered}
T_{t}^{t}=T_{r}^{r}=\rho_{q}, \\
T_{\varphi}^{\varphi}=T_{\theta}^{\theta}=-\frac{1}{2} \rho_{q}(3 \omega+1),
\end{gathered}
$$

where $\omega$ is the quintessence state parameter and $\rho_{q}$ is the density of quintessence matter given by

$$
\rho_{q}=-\frac{\alpha}{2} \frac{3 \omega}{r^{3(\omega+1)}}
$$

where $\alpha$ is a constant called normalization factor. Solution of the Einstein field equations with energy momentum tensor given by equs. (1) and (2) leads to the following metric

$$
d s^{2}=f(r) d t^{2}-\frac{d r^{2}}{f(r)}-r^{2}\left(d \theta^{2}+\sin ^{2} \theta d \varphi^{2}\right)
$$

where $f(r)$ is given by

$$
f(r)=1-\frac{2 m}{r}-\frac{\alpha}{r^{3 \omega+1}}
$$


where $m$ is the black hole mass. For the existence of cosmological horizon we have the following constraint over the allowable values of $\omega$

$$
-1<\omega<-\frac{1}{3}
$$

The scalar curvature is given as

$$
R=2 T_{\lambda}^{\lambda}=3 \alpha \omega \frac{(1-3 \omega)}{r^{3(\omega+1)}} .
$$

It is clear from equ. (7) that there is a singularity at $r=0$ if $\omega \neq-1,0, \frac{1}{3}$.

For $\omega=-\frac{2}{3}$ the metric (4) takes the form

$$
d s^{2}=\left(1-\frac{2 m}{r}-\alpha r\right) d t^{2}-\frac{d r^{2}}{\left(1-\frac{2 m}{r}-\alpha r\right)}-r^{2}\left(d \theta^{2}+\sin ^{2} \theta d \varphi^{2}\right)
$$

The horizons are at

$$
r_{ \pm}=\frac{1 \pm \sqrt{1-8 \alpha m}}{2 \alpha}
$$

Here $r_{-}$and $r_{+}$are black hole and cosmological horizons respectively. Clearly the horizons are possible, when

$$
m<\frac{1}{8 \alpha}
$$

which gives an upper limit on mass, $m$, for a given value of $\alpha$. 




Fig. 1. The figure depicts the relation between $r$ and $f(r)$ for $\alpha=0.15$ and $m=0.5$.

\section{Non-singular compactified coordinates for SHQ}

In order to examine the geometrical and physical features of the SHQ, we need to construct the coordinates that can smoothly cross the horizons. The technique for resolving the problem of unsatisfactory coordinates is to investigate and explore the spacetime with geodesics, which being coordinate independent will not be influenced by coordinate validity boundaries. In this regard we use the worldlines of radially moving photons as considered by Eddington-Finkelstein [8, 9]. For metric (8) the radial null geodesics are given by

$$
t= \pm\left\{\ln \left|\frac{r}{r_{+}}-1\right|^{\frac{r_{+}}{r_{+}-r_{-}}}-\ln \left|\frac{r}{r_{-}}-1\right|^{\frac{r_{-}}{r_{+} r_{-}}}+\text {constant }\right\}
$$

where plus/minus sign corresponds to the outgoing/incoming photons.

For the construction of Eddington-Finkelstein like coordinates, we define the constants of integration appearing in equ. (11) as new coordinates and label them by $p$ and $q$. Thus, the coordinate transformations are given by 


$$
\begin{aligned}
& p=t+\left\{\ln \left|\frac{r}{r_{+}}-1\right|^{\frac{r_{+}}{r_{+}-r_{-}}}-\ln \left|\frac{r}{r_{-}}-1\right|^{\frac{r_{-}}{r_{+}-r_{-}}}\right\}, \\
& q=t-\left\{\ln \left|\frac{r}{r_{+}}-1\right|^{\frac{r_{+}}{r_{+}-r_{-}}}-\ln \left|\frac{r}{r_{-}}-1\right|^{\frac{r_{-}}{r_{+}-r_{-}}}\right\} .
\end{aligned}
$$

For the construction of Kruskal-Szekeres like coordinates, we use the following transformations

$$
\tilde{p}=\lambda \exp \left(\frac{p}{\beta}\right), \quad \tilde{q}=-\lambda \exp \left(\frac{-q}{\beta}\right)
$$

To get the usual form of the mertic we define new variables $u$ and $v$, which are spacelike and timelike respectively, and are given by

$$
V=\frac{\tilde{p}+\tilde{q}}{2}, \quad u=\frac{\tilde{p}-\tilde{q}}{2}
$$

Using equs. (12)-(14) in equ. (15) we have

$$
\begin{aligned}
& V=\lambda \sinh \left(\frac{t}{\beta}\right)\left|\frac{r}{r_{+}}-1\right|^{\frac{r_{+}}{\beta\left(r_{+}-r_{-}\right)}}\left|\frac{r}{r_{-}}-1\right|^{\frac{-r_{-}}{\beta\left(r_{+}-r_{-}\right)}}, \\
& u=\lambda \cosh \left(\frac{t}{\beta}\right)\left|\frac{r}{r_{+}}-1\right|^{\frac{r_{+}}{\beta\left(r_{+}-r_{-}\right)}}\left|\frac{r}{r}-1\right|^{\frac{-r_{-}}{\beta\left(r_{+}-r_{-}\right)}} .
\end{aligned}
$$

The coordinates given by equs.(16) and (17) are singular at $r=r_{ \pm}$. In order to eliminate 
the singularity we need to cancel the factors containing $\left(1-\frac{r}{r_{-}}\right)$and $\left(1-\frac{r}{r_{+}}\right)$, but in any way both can not be canceled simultaneously. So to keep the coordinates regular at $r=r_{+}$we take

$$
\beta=\beta_{+}=\frac{2 r_{+}}{r_{+}-r_{-}}, \quad \lambda=\lambda_{+},
$$

and for coordinates to be regular at $r=r_{-}$we take

$$
\beta=\beta_{-}=\frac{-2 r_{-}}{r_{+}-r_{-}}, \quad \lambda=\lambda_{-}
$$

The values of $\lambda_{ \pm}$are chosen so that at a point $r$, between $r_{+}$and $r_{-}$hypersurfaces get matched properly in $\left(v_{-}, u_{-}\right)$and $\left(v_{+}, u_{+}\right)$coordinates. Since the coordinates $\left(v_{-}, u_{-}\right)$are regular at $r=r_{-}$they are used for the region in which $0<r<r_{+}$. Similarly as the coordinates $\left(v_{+}, u_{+}\right)$ are regular at $r=r_{+}$, so they are used for $r_{-}<r<\infty$. Here $r=r_{ \pm}$correspond to $v_{+}=u_{+}=0$ and $v_{-}=u_{-}=0$ respectively. The implicit relation between $u, v$ and $r$ is

$$
\begin{aligned}
& u_{+}^{2}-V_{+}^{2}=\lambda_{+}^{2}\left|\frac{r}{r_{+}}-1\right|^{\frac{2 r_{+}}{\beta_{+}\left(r_{+}-r_{-}\right)}}\left|\frac{r}{r}-1\right|^{\frac{2 r_{-}}{\beta_{+}\left(r_{+}-r_{-}\right)}}, \\
& u_{-}^{2}-V_{-}^{2}=\lambda_{-}^{2}\left|\frac{r}{r_{+}}-1\right|^{\frac{2 r_{+}}{\beta_{-}\left(r_{+}-r_{-}\right)}}\left|\frac{r}{r}-1\right|^{\frac{2 r_{-}}{\beta_{-}\left(r_{+}-r_{-}\right)}} .
\end{aligned}
$$

To get the compactified coordinates for SHQ metric we use the coordinate transformations as

$$
\begin{gathered}
\psi=\tan ^{-1}(V+u)+\tan ^{-1}(v-u) \\
\xi=\tan ^{-1}(v+u)-\tan ^{-1}(v-u) . \\
478
\end{gathered}
$$




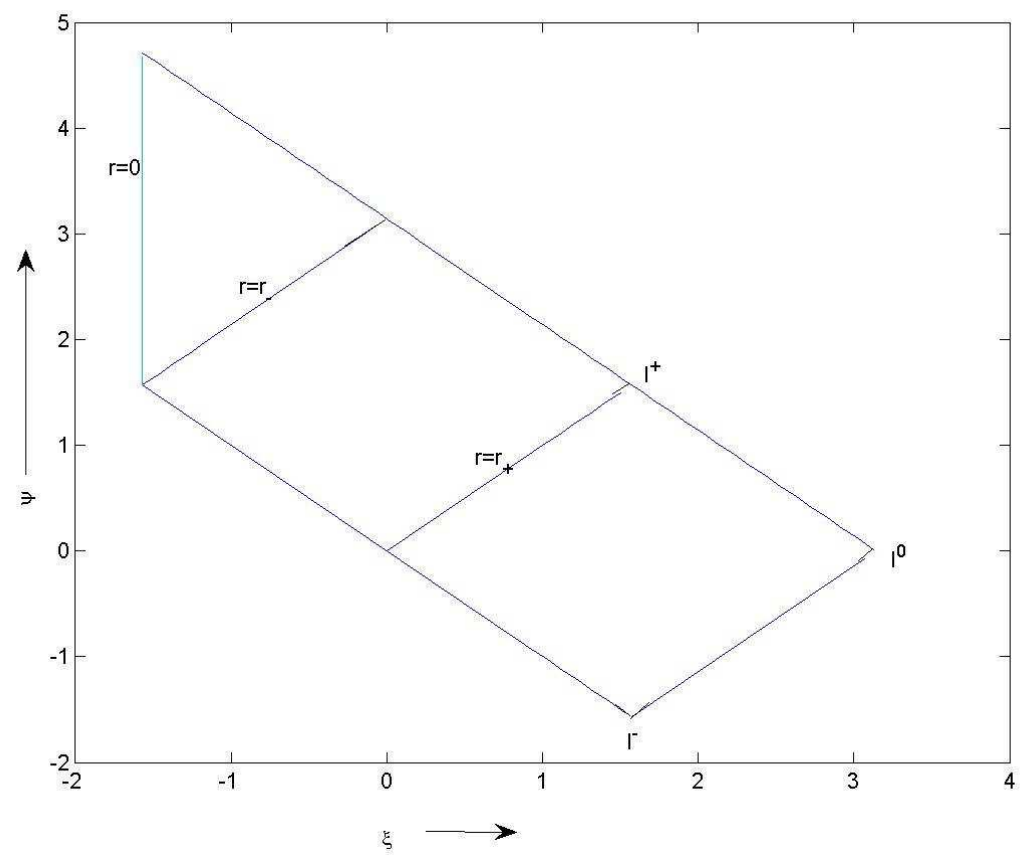

Fig. 2. Carter-Penrose diagram of the SHQ spacetime. Note that the essential singularity here is timelike.

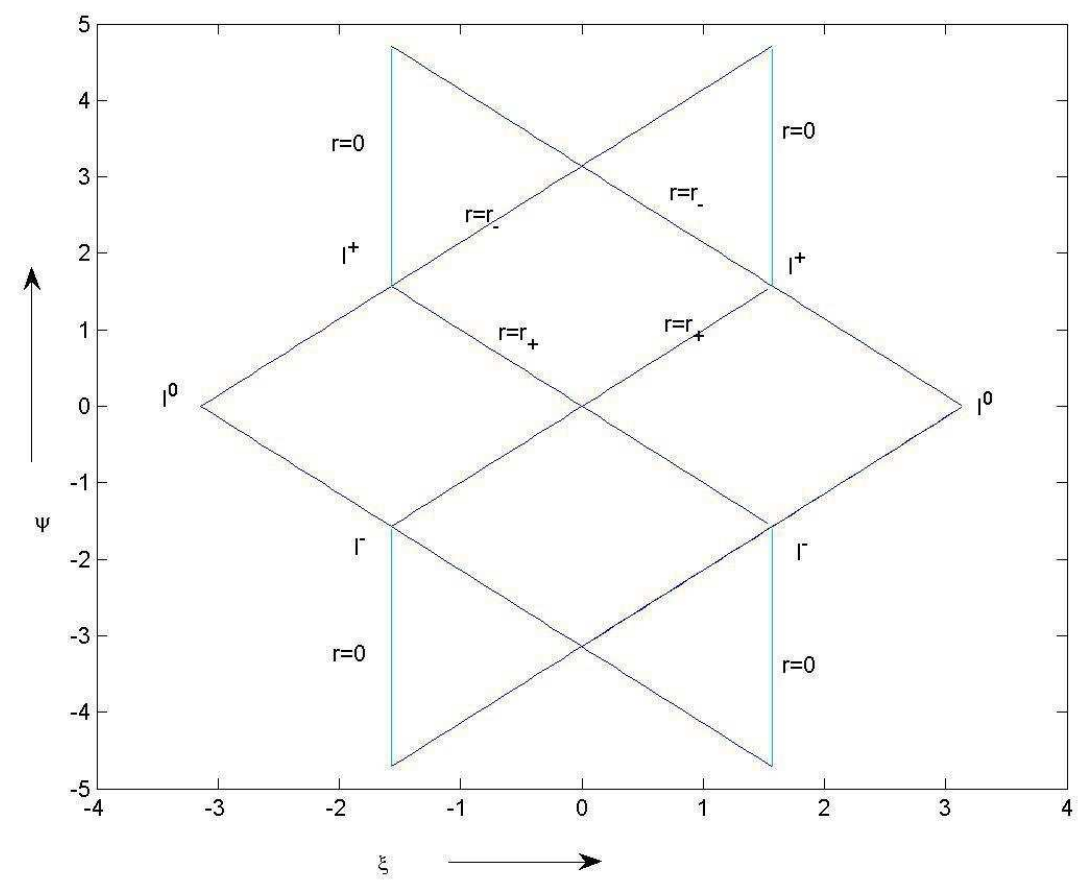

Fig. 3. Maximally extended Carter-Penrose diagram of the SHQ spacetime. Note that as the essential singularity is timelike, so a time like curve can enter the new universes by passing through the two coordinate singularities. 
The implicit relation between $\psi, \xi$ and $r$ is given as

$$
u^{2}-V^{2}=\lambda^{2}\left|\frac{r}{r_{+}}-1\right|^{\frac{2 r_{+}}{\beta\left(r_{+}-r_{-}\right)}}\left|\frac{r}{r_{-}}-1\right|^{\frac{2 r_{-}}{\beta\left(r_{+}-r_{-}\right)}}=-\tan \frac{\psi+\xi}{2} \tan \frac{\psi-\xi}{2} .
$$

\section{Foliation of SHQ by hypersurfaces of constant mean extrinsic curvature}

In order to obtain the constant mean extrinsic curvature hypersurfaces for SHQ we follow the procedure of Brill et. al. [4] and obtain the following ordinary differential equation in KruskalSzekeres coordinates

$$
\frac{d v}{d u}=\frac{A v+E u}{A u+E V}
$$

with

$$
E(r)=H-\frac{K r^{3}}{3}, \quad A^{2}(r)=E^{2}(r)+r^{3}\left(r-r_{+}\right)\left(r-r_{-}\right)
$$

In compactified Kruskal-Szekeres coordinates equ. (25) is given as

$$
\frac{d \psi}{d \xi}=\frac{A \sin \psi \cos \xi+E \sin \xi \cos \psi}{A \sin \xi \cos \psi+E \sin \psi \cos \xi}
$$

In order to obtain the foliation we follow the similar procedure as is given in reference [5]. For a specific value of $K$ we require that $A=0$ at $\xi=0$ in equ. (27). This requirement provides a relation between initial value of $r$ and $H$ given as

$$
H=\frac{K r_{i}^{3}}{3} \pm \sqrt{r_{i}^{3}\left(r_{i}-r_{+}\right)\left(r_{i}-r_{-}\right)}
$$

where $r_{i}$ is the initially selected value of $r$. For each choice of $K$ we get two choices of $H$, of which 
we select the one having sign opposite to that of the sign of $K$. In this way we get an initial value of $\psi$ and call it as $\psi_{*}$. Also for $K \rightarrow-K$ we get the hypersurfaces by taking $H \rightarrow-H$. So on both $\xi$ and $\psi$ axis we have the reflection symmetry.

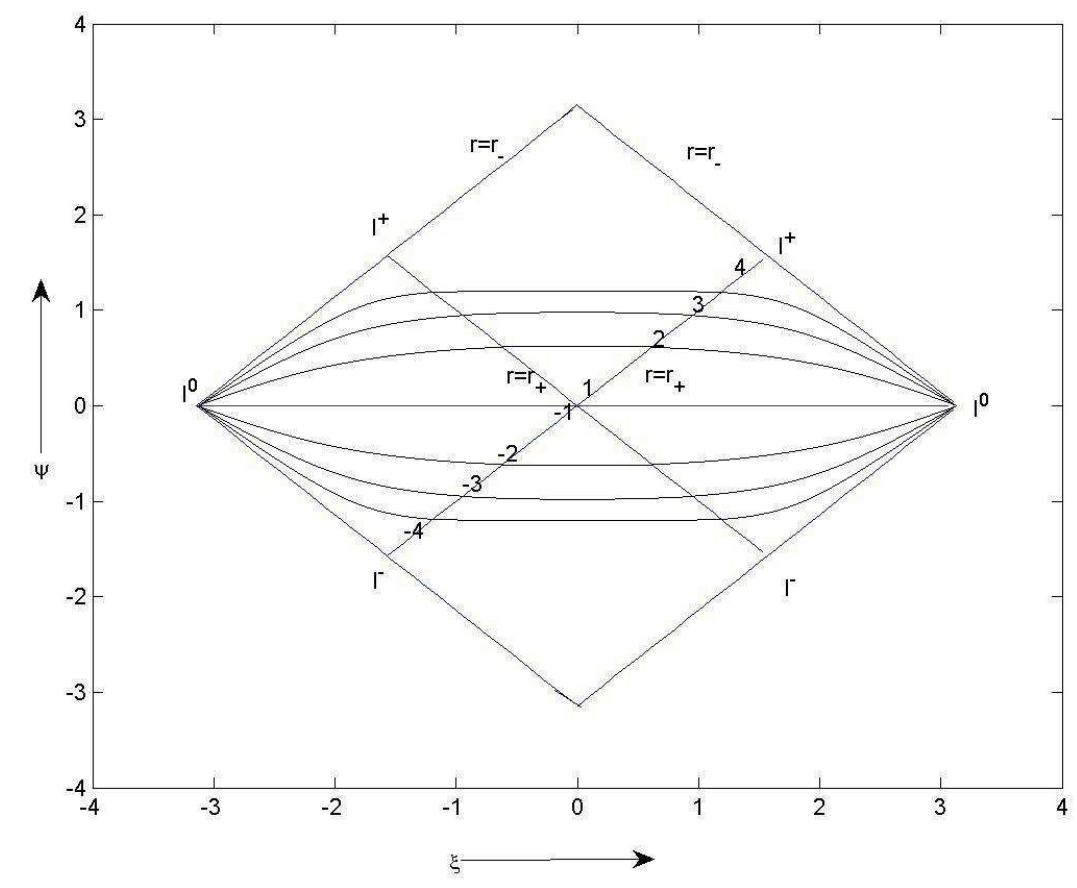

Fig. 4. Foliation of the SHQ spacetime in Carter-Penrose diagram by $K$-surfaces. Few spacelike hypersurfaces for $K=0, \pm 0.00001, \pm 0.005, \pm 0.015$ are shown.

\begin{tabular}{|c|c|c|c|c|}
\hline NO. & $K$ & $r_{i}$ & $H$ & $\psi_{*}$ \\
\hline 1 & 0 & $r_{+}$ & 0 & 0 \\
\hline \pm 2 & \pm 0.00001 & 7.2 & $\mp 23.6077$ & \pm 0.6257 \\
\hline \pm 2 & \pm 0.005 & 6.8 & $\mp 33.8600$ & \pm 0.9786 \\
\hline \pm 2 & \pm 0.015 & 6.4 & $\mp 37.3670$ & \pm 1.1976 \\
\hline
\end{tabular}

Table 1 . The mean extrinsic curvature, $K$, initial value, $r_{i}$, of $r$, constant $H$, and initial value of $\psi$, labelled as $\psi_{*}$ are given for seven different $K$-surfaces. 


\section{Conclusions}

In this paper, we have considered the Schwarzschild black hole surrounded by quintessence (SHQ) for the case when there are two horizons. This case is similar to the usual ReissnerNordstrom black hole (RN). Following the similar procedure as adopted for the RN black hole we have constructed the non singular Kruskal-Szekeres like coordinates for the SHQ. We have also presented the Carter-Penrose diagram for this geometry. A foliation of the Carter-Penrose diagram by hypersurfaces having constant mean extrinsic curvature is obtained. Like foliation of the RN black hole [8] here the hypersurfaces also foliate the geometry upto the inner horizon.

\section{References}

1. Hobson M.P., Efstathiou G.P., Lasenby A.N. (2006). General relativity: an introduction for physicists. Cambridge: Cambridge University Press.

2. Lichnerowicz A. (1944). Lintegration des equations de la gravitation relativiste et le probleme des n corps. J. Math. Pures Appl, 23(37), 4.

3. York Jr. J.W. (1971). Gravitational degrees of freedom and the initial-value problem. Physical Review Letters, 26(26):1656.

4. Brill D.R., Cavallo J.M., Isenberg J.A. (1980). K-surfaces in the schwarzschild space-time and the construction of lattice cosmologies. Journal of Mathematical Physics, 21(12): 2789-2796.

5. Pervez A., Qadir A., Siddiqui A.A. (1995). Foliation by constant-mean-curvature hypersurfaces of the schwarzschild spacetime. Physical Review, D 51(8):4598.

6. Qadir A., Siddiqui A.A. (1999). K-slicing the schwarzschild and the reissner-nordstrom spacetimes. Journal of Mathematical Physics, 40(11):5883-5889.

7. Kiselev V.V. (2003). Quintessence and black holes. Classical and Quantum Gravity, 20(6): 1187.

8. Eddington A.S. (1924). A comparison of Whitehead's and Einstein's formulae. Nature, 113:192.

9. Finkelstein D. (1958). Past-future asymmetry of the gravitational field of a point particle. Physical Review, 110(4):965. 